\title{
O uso de tecnologias de informação e comunicação (TIC) em experiências de pós-graduação sobre promoção da saúde no Brasil e na Costa Rica
}

\author{
The use of information technology in the postgraduate course in the health \\ promotion in Brazil and Costa Rica
}

\section{Ana Claudia Camargo Gonçalves Germani ${ }^{1}$, Alfredo Almeida Pina de Oliveira ${ }^{2}$, Hellen Pimentel Ferreira ${ }^{3}$, Angela Cristina Yano ${ }^{4}$, Gabriela Murillo Sancho ${ }^{5}$}

Germani ACCG, Oliveira AAP, Ferreira HP, Yano AC, Sancho GM. O uso de tecnologias de informação e comunicação (TIC) em experiências de pós-graduação sobre promoção da saúde no Brasil e na Costa Rica / The use of information technology in the postgraduate course in the health promotion in Brazil and Costa Rica. Rev Med (São Paulo). 2013 abr.-jun.;92(2):97-103.

\begin{abstract}
RESUMO: As Conferências Internacionais de promoção da saúde ressaltam a necessidade de reorientação dos serviços como campo de ação da área. Em especial a Carta de Bangkok menciona as Tecnologias de Informação e Comunicação (TIC). Nesta direção, o ensino baseado em TIC surge como forma de fortalecer o aprender a aprender, também na área de promoção da saúde, tema ainda latente nas graduações. Objetivo: Descrever o uso das TIC em cursos de pós-graduação com foco na promoção da saúde. Método: Trata-se de uma análise descritiva das abordagens de ensino apoiadas em TIC em três experiências. A primeira, na modalidade de atualização/especialização (lato senso), é parte das ações de extensão do Departamento de Clínica Médica e vem incorporando as TIC ao longo de seus 10 anos de existência. A segunda experiência aborda uma disciplina de pós-graduação stricto senso do Programa de Saúde Coletiva, sob responsabilidade do Departamento de Medicina Preventiva. Por fim, é apresentada a disciplina de pós-graduação também stricto senso de Enfermagem da Faculdade de Medicina na Universidade da Costa Rica. Resultados: Apresentam-se quadros sintéticos sobre o uso de diferentes TIC bem como os objetivos pedagógicos pretendidos e a natureza da atividade. Conclusão: $\mathrm{O}$ atual uso das TIC evidencia a oportunidade de espaço e estímulo para construção e re-construção do conhecimento em Educação e Promoção da Saúde. Reconhecer as potencialidades e os limites do uso de TIC contribui para a formação interprofissional de promotores da saúde com ênfase em uma educação transformadora.
\end{abstract}

DESCRITORES: Promoção da saúde; Educação em saúde; Tecnologia educacional; Tecnologia da informação; Relações interprofissionais; Educação de pós-graduação; Brasil; Costa Rica.
ABSTRACT: Global Conferences on Health Promotion underscore the need for reorientation of services as a field of action in the area. In particular the Bangkok Charter mentions the Information and Communication Technologies (ICT). In this sense, ICT-based teaching emerges as a way to strengthen learning how to learn, also in the area of health promotion, theme still latent into graduation's health course. Objective: To describe the use of ICT in post-graduate focusing on health promotion. Method: This is a descriptive analysis of teaching approaches supported by ICT in three experiments. The first, in the form of update / specialization (sensu lato), is part of the activities of the department of internal medicine and has been incorporating ICT throughout its 10 years of existence. The second experiment deals with a post-graduate course in the strict sense of the public health program, under the responsibility of the preventive medicine department. Finally, we present the discipline graduate also strict sense of Nursing, Faculty of Medicine at the University of Costa Rica. Results: We present summary tables on the use of different ICT as well as the desired educational objectives and the nature of the activity. Conclusion: The current use of ICT highlights the opportunity and incentive for space construction and re-construction of knowledge in Health Promotion. Recognize the potential and limits of the use of ICT contributes to interprofessional education of health promoters with emphasis in a transformative education.

KEYWORDS: Health promotion; Health education; Educational technology; Information technology; Interprofessional relations; Education, graduate; Brazil; Costa Rica.

Trabalho realizado na Faculdade de Medicina da Universidade de São Paulo - FMUSP e Escola de Enfermagem da Faculdade de Medicina - Universidade da Costa Rica.

1. Médica. Professora Doutora do Departamento de Medicina Preventiva da Faculdade de Medicina da Universidade de São Paulo - FMUSP. E-mail: accggermani@usp.br

2. Enfermeiro. Doutorando e Mestre em Ciências da Saúde pelo Programa de Pós Graduação em Enfermagem em Saúde Coletiva da Escola de Enfermagem da Universidade de São Paulo - EEUSP. Docente da Faculdade de Campo Limpo Paulista (FACCAMP). E-mail:aapo_enf@ yahoo.com.br

3. Psicóloga. Mestre em Ciências pela Faculdade de Medicina da Universidade de São Paulo - FMUSP. E-mail: hellenhl2@hotmail.com

4. Médica. Especialista em Promoção da Saúde pela Faculdade de Medicina da Universidade de São Paulo - FMUSP. E-mail: angelayano@ hotmail.com

5. Educadora e psicopedagoga, pós-graduanda do Mestrado em Saúde Pública com ênfase em gestão de saúde. Docente e pesquisadora na Escola de Saúde Pública e docente na Escola de Enfermagem, Faculdade de Medicina, Universidade de Costa Rica. E-mail: gaby_2000m@ yahoo.com

Endereço para correspondência: Ana Claudia C. G. Germani. FMUSP - Depto. de Medicina Preventiva. Av. Dr. Arnaldo, $455,2^{\circ}$ andar, sala 2221. São Paulo, SP - Brasil. CEP 01246-903. E-mail: accggermani@usp.br 


\section{INTRODUÇÃO}

$\mathbf{F}$ rente ao aumento expressivo da circulação e armazenamento de informações na sociedade moderna, a Comissão Internacional sobre a Educação para o Século XXI da UNESCO (United Nations Educational, Scientific and Cultural Organization) aponta que a educação deve assumir dois grandes objetivos. $\mathrm{O}$ primeiro deles é a transmissão de forma maciça e eficaz de cada vez mais saberes, ou seja, cabe à educação fornecer um mapa do mundo atual. Ao mesmo tempo, é essencial que o processo de ensino-aprendizagem assinale as referências que impeçam as pessoas de ficarem submersas nas ondas de informações (algumas efêmeras) que invadem os espaços públicos e privados, mantendo a analogia, a educação deve ser uma bússola que permita a navegação através do mapa ${ }^{1}$ (Delors, 2003).

A mesma Comissão propõe quatro domínios para a educação, quatro formas de aprendizagem fundamentais ao longo da vida de um indivíduo: aprender a aprender (aprender para conhecer, exercitando a atenção, a memória e o pensamento), aprender a fazer (pôr em prática os conhecimentos de forma a estar apto a enfrentar numerosas situações de trabalho), aprender a conviver com os outros (desenvolver a compreensão do outro e a percepção das interdependências, realizar projetos comuns e preparar-se para gerir conflitos) e aprender a ser (agir com autonomia, discernimento e responsabilidade pessoal).

A evolução contínua e extremamente veloz dos conhecimentos cria a necessidade de permanente atualização do homem. Pierre Lévy ${ }^{1}$, filósofo da informação que se ocupa em estudar as interações entre a internet e a sociedade, aponta que "pela primeira vez na história da humanidade, a maioria das competências adquiridas por uma pessoa no começo de seu percurso profissional serão obsoletas no fim de sua carreira". Ebert $^{2}$, em publicação nacional, coloca a exigência do mercado por profissionais com mais qualificação, novas competências e habilidades, obrigando-os a um aprendizado contínuo.

As Tecnologias de Informação e Comunicação (TIC) são um conjunto de recursos tecnológicos e computacionais dedicados ao armazenamento, processamento e comunicação da informação. Diante de tecnologias unificadoras, que ultrapassam fronteiras geográficas, culturas e fusos horários, podemos dizer que hoje o acesso à educação não tem tempo ou local fixo. A comunicação de informações armazenadas e acessadas em tempos diferentes passa a ocorrer sem que seja perdida a interatividade ${ }^{3}$.

Atualmente, verifica-se grande diversidade das TIC usadas para a Educação. Vale mencionar que estes recursos podem envolver a comunicação assíncrona ou síncrona. Na primeira, as pessoas podem aprender por meio de uma rede de computadores em qualquer hora e em qualquer lugar, sem a participação simultânea de todos os envolvidos no processo de ensino-aprendizagem. Como exemplo de ferramenta de cooperação assíncrona, pode-se citar o correio eletrônico. Já na comunicação síncrona, as pessoas estabelecem comunicação de forma simultânea, contando com outras ferramentas de cooperação (como chats).

A aplicação das TIC para incremento da aprendizagem em equipes interdisciplinares/interprofissionais é descrita tanto em experiências durante a graduação e com profissionais já formados ${ }^{4,5,6}$.

Tendo em vista a continuidade da formação após graduação, a Organização Pan-Americana de Saúde (OPAS) já discutia em 1991 o processo educativo nos serviços de saúde, sendo o desenvolvimento de mecanismos para a educação permanente a questão central das reflexões. O material enfatizava programas educativos que valorizassem o trabalhador da saúde como sujeito e fossem estruturados a partir de um recorte das necessidades concretas, com posterior organização e estruturação dos conhecimentos existentes e que acima de tudo permitissem a crítica e transformação da prática ${ }^{7}$.

No campo da Promoção da Saúde, as Conferências Internacionais reforçam a necessidade de uma reorientação dos serviços, que inclui a formação dos profissionais de saúde ${ }^{8}$. A Carta de Bangkok, publicada em 2005, tem como foco a globalização e menciona a melhoria das TIC como oportunidade para o enfrentamento dos determinantes sociais ${ }^{9}$.

Para exemplificar, ainda nas considerações iniciais, a Organização Pan-Americana de Saúde (OPAS) oferece o curso virtual on-line "Promoción de la salud: conceptos, herramientas y estratégias" (http://cursostaff. campusvirtualsp.org/course/view.php?id=14) composto por três módulos, de acesso livre. Entretanto, apesar do número crescente de experiências semelhantes de formação de promotores da saúde, o apoio das TIC na formação de promotores de saúde é um tema pouco sistematizado.

\section{OBJETIVO}

Descrever o uso das TIC em dois cursos de pósgraduação com foco na promoção da saúde.

\section{MÉTODO}

Seguindo recomendações sobre a pesquisa em educação médica, optou-se por responder as perguntas do presente estudo tendo como marco teórico o modelo dos 3Ps: Presage-Process-Product, sintetizado no Quadro $1^{10,11}$. 
QUADRO 1. Modelo dos 3Ps Presage-Process-Product

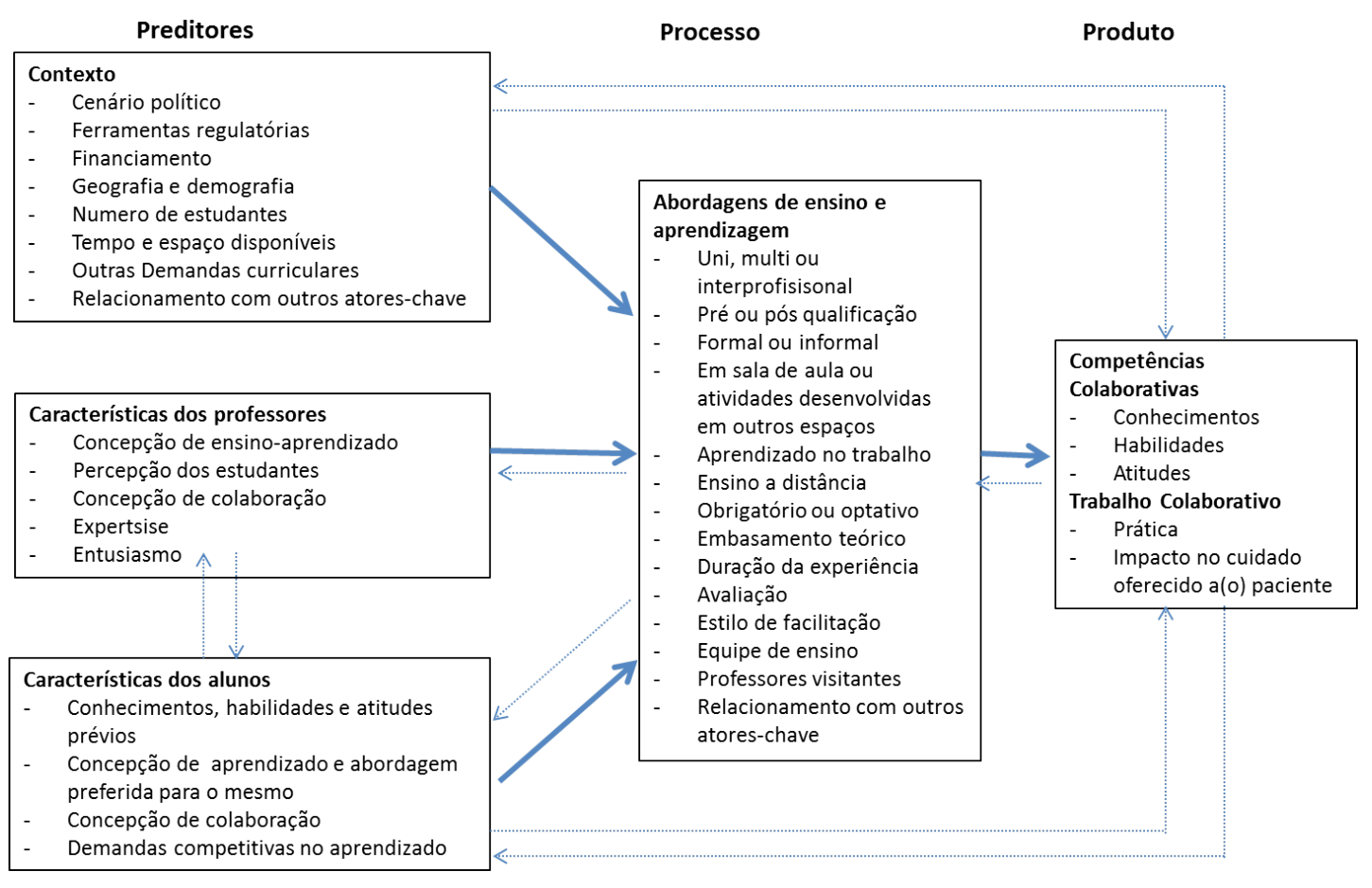

Trata-se de uma análise descritiva das abordagens de ensino apoiadas em TIC, ou seja, do segundo P referente ao processo de ensino. Foram utilizadas como categorias de análise: duração da experiência, utilização das TIC pelos professores em ambiente presencial ou virtual, uso obrigatório ou optativo por parte dos estudantes e caráter uni, multi ou interprofissional da abordagem.

Para tanto, foram escolhidas três experiências em cursos de pós-graduação no Brasil e na Costa Rica: duas delas oferecidas pela Faculdade de Medicina da Universidade de São Paulo voltadas à formação de promotores de saúde em um curso de atualização/especialização (lato sensu) e em uma disciplina vinculada ao Departamento de Medicina Preventiva (stricto sensu); e outra desenvolvida na Universidade da Costa Rica, que trabalha a educação em saúde com foco na criança e no adolescente.

Promoção da Saúde e Educação em Saúde não são sinônimos, embora tenham semelhanças e diferenças entre si. No cenário internacional, alguns autores usam os termos promoção da saúde e educação em saúde como se fossem intercambiáveis, enquanto outros enfatizam as diferenças de abordagem, princípios e efetividade de cada $\mathrm{um}^{12}$.

Entende-se neste artigo que a promoção da saúde envolve a capacitação da comunidade e desta forma, uma das principais estratégias consiste na educação em saúde, que deve estar centrada no desenvolvimento de práticas participativas, no enfrentamento de necessidades de saúde, no incentivo e no respeito pelos sujeitos envolvidos e na proposição de soluções criativas. Entende-se, portanto, que cada pessoa constrói seu próprio conhecimento, a partir de experiências pessoais e encontros sociais.

Contudo, é importante reforçar que a promoção incorpora e utiliza outras estratégias já mencionadas na Carta de Ottawa, como a mediação intersetorial de interesses para a concretização de ações e a advocacia para a promoção da saúde, constituída por ações de indivíduos ou de grupos organizados - os chamados atores sociais que procuram influir sobre autoridades e sobre indivíduos, para reivindicar direitos na área da saúde e garantir a equidade.

\section{Curso de atualização-especialização: Práticas de Promoção da Saúde}

Em 2004, professores, pesquisadores e outros colaboradores do departamento de Clínica Médica da FMUSP, criaram o Curso de atualização e especialização intitulado "Práticas de Promoção da Saúde". Destinado a profissionais da área da saúde e outros profissionais envolvidos em programas de qualidade de vida e promoção da saúde, o mencionado curso ainda hoje objetiva capacitar os participantes no campo da promoção da 
saúde, com conteúdo científico atualizado e sua aplicação prática, destacando programas de atuação em empresas e instituições de saúde pública e privada. Anualmente, são oferecidas 80 vagas.

Desde então, o curso oferece o conteúdo distribuído em cinco módulos presenciais: prática clínica, comportamento, gestão de doenças crônicas, saúde do trabalhador/comunidade e gestão da promoção da saúde. Cada módulo é composto por aulas teóricas (atualização) e práticas (especialização) contando com metodologias ativas e incorporação de TIC em sala de aula e propostas de atividades em ambiente virtual. O corpo docente conta com professores dos cursos de Medicina e Enfermagem, além de convidados externos, alguns da iniciativa privada.

Levantamento feito entre 2006 e 2008, mostrou que a média de idade dos participantes era de 36 anos (desvio padrão 9 anos), com o predomínio de mulheres e de profissionais ligados à área da saúde, sobretudo da enfermagem e medicina. A maior parte dos alunos possuía 10 anos ou mais de formado.

A cada ano, a seleção das tecnologias digitais utilizadas no curso baseia-se nos objetivos pedagógicos propostos a cada módulo, na coordenação pedagógica do curso, nas estratégias didáticas de cada professor convidado e na avaliação feita pelos participantes do ano anterior.

Disciplina Prevenção de agravos, Promoção da Saúde e Redução de Vulnerabilidade: evolução histórica dos conceitos e oportunidades de práticas interprofissionais

Oferecida há dois anos, a disciplina faz parte do programa de pós-graduação do Programa de Saúde Coletiva do Departamento de Medicina Preventiva da Faculdade de Medicina da USP (FMUSP). Tem como objetivo, ao longo de seis aulas presenciais, discutir os pressupostos teóricos e implicações práticas no processo de emergência histórica e desenvolvimento dos conceitos de História Natural da Doença/Níveis de Prevenção, Promoção da Saúde e Vulnerabilidade.

A coordenação conta com dois docentes médicos e um historiador da FMUSP. O corpo docente conta ainda com convidados da Escola de Enfermagem e do Departamento de Terapia Ocupacional da mesma Universidade. Participam da disciplina pós-graduandos de cursos variados (medicina, enfermagem, terapia ocupacional, psicologia, ciências sociais entre outros).

A carga horária prevê atividades presenciais e realização de leituras, que envolvem o contato com TIC, sobretudo de forma assíncrona. Ao final das aulas, há a avaliação dos participantes e avaliação do programa pelos docentes e discentes.

\section{Disciplina de pós-graduação em enfermagem: "Educação pela saúde na infância e adolescência"}

Oferecida desde 2005, faz parte do programa de Pós-Graduação em Enfermagem Pediátrica da Escola de Enfermagem da Universidade de Costa Rica e recebe graduados em enfermagem, que trabalham em hospitais e clínicas públicas ou privadas.

A disciplina tem como objetivos: 1) Desenvolver uma visão complexa de educação em saúde a partir de abordagens atuais; 2) Desenvolver ações coordenadas de educação em saúde para crianças e adolescentes, a partir da perspectiva da implementação do projeto; e 3) Avaliar as ações coordenadas realizadas com crianças e adolescentes, provenientes dos orçamentos da avaliação. Tais objetivos buscam o desenvolvimento de competências relacionadas a educação em saúde, essenciais ao promotor da saúde.

Metodologicamente, são propostos: e-portfolios, desenvolvimento de fóruns participativos (presencial ou virtual) e mapeamento de conceitos. São previstas atividades presenciais e a distância.

Ao final da disciplina, avalia-se o impacto do desempenho do portfólio na vida profissional de cada estudante.

\section{RESULTADOS}

Os três cursos analisados utilizaram doze diferentes tipos de TIC, sendo que o uso de vídeos e de mapas conceituais aparece em duas das experiências estudadas. Tais ferramentas foram organizadas em três quadros (Quadros 2, 3 e 4), de acordo com a experiência acadêmica.

Observam-se mudanças na velocidade de incorporação e também na manutenção da utilização de tais dispositivos ao longo do tempo, em duas das experiências acadêmicas analisadas.

A análise das finalidades da abordagem de ensino mostra que cinco $(41,6 \%)$ das ferramentas demandam a participação efetiva dos estudantes: grupo de e-mails, palavras-cruzadas, mapas conceituais, sala de aula virtual e e-portfolios. O acesso ao material das aulas e referências bibliográficas foi oferecido em outras cinco ferramentas, que incluíram um site para hospedagem do material, a seleção de uma webliografia, um blog como quadro de avisos, o Moodle ${ }^{\circledR}$ e o Dropbox ${ }^{\circledR}$, sendo os dois últimos espaços abertos à contribuição dos participantes.

Duas TIC (Prezi ${ }^{\circledR}$ e a nuvem de palavras criada no Wordle ${ }^{\circledR}$ ) foram usadas apenas em sala de aula. Cabe pontuar também que a maior parte das tecnologias utilizadas tinha caráter obrigatório. 
QUADRO 2. Tecnologias de Informação e Comunicação usadas como materiais didáticos desenvolvidos no curso de atualização/ especialização de Práticas de Promoção da Saúde, entre 2004 a 2013

\begin{tabular}{|c|c|c|c|c|}
\hline Ano & TIC & Finalidade da abordagem de ensino & $\begin{array}{l}\text { Ambiente } \\
\text { de uso }\end{array}$ & $\begin{array}{l}\text { Natureza da } \\
\text { atividade }\end{array}$ \\
\hline $\begin{array}{l}2004 \text { a } \\
2009\end{array}$ & $\begin{array}{l}\text { Acesso on-line ao ma- } \\
\text { terial de aula e leituras } \\
\text { sugeridas }\end{array}$ & Oferecer formas de contato com o conteúdo teórico & Virtual & Obrigatória \\
\hline $\begin{array}{l}2006 \mathrm{a} \\
2008\end{array}$ & $\begin{array}{l}\text { Grupo de } e \text {-mail, } \\
\text { plataforma gmail }{ }^{\circledR}\end{array}$ & $\begin{array}{l}\text { Criar espaço assíncrono para discussões sobre tópicos das } \\
\text { aulas presenciais, além da troca de experiências profissio- } \\
\text { nais e pessoais. Em 2008, houve a contribuição de uma } \\
\text { tutora na mediação das conversas. }\end{array}$ & Virtual & Optativa \\
\hline $\begin{array}{l}2006 \text { a } \\
2008\end{array}$ & Blog & $\begin{array}{l}\text { Criar um quadro de avisos sobre andamento do curso e } \\
\text { permitir o acesso a webliografia. }\end{array}$ & Virtual & Obrigatória \\
\hline $\begin{array}{l}2007 \text { e } \\
2008\end{array}$ & $\begin{array}{l}\text { Organização de } W e- \\
\text { bliografia }\end{array}$ & $\begin{array}{l}\text { Selecionar a literatura on-line relevante e torná-la acessí- } \\
\text { vel aos profissionais de saúde, possibilitando a aquisição } \\
\text { de conhecimentos novos ou rápidas atualizações em } \\
\text { promoção da saúde e prevenção de doenças. }\end{array}$ & Virtual & Obrigatória \\
\hline $\begin{array}{l}2007 \text { e } \\
2008\end{array}$ & $\begin{array}{l}\text { Palavras Cruzadas, } \\
\text { criada com o software } \\
\text { livre Hotpotatoes } \AA\end{array}$ & $\begin{array}{l}\text { Estimular a auto avaliação do estudante relacionada à re- } \\
\text { tenção de conceitos-marcadores. (preenchimento na web } \\
\text { ou após download) }\end{array}$ & Virtual & Optativa \\
\hline $\begin{array}{l}2006 \text { até } \\
2013\end{array}$ & Vídeos do YouTube ${ }^{\circledR}$ & $\begin{array}{l}\text { Seleção de curtas-metragens feita pelos professores, para } \\
\text { reflexão sobre a temática da aula }\end{array}$ & $\begin{array}{l}\text { Em sala de } \\
\text { aula }\end{array}$ & $\begin{array}{l}\text { Desenvolvida } \\
\text { pelo professor }\end{array}$ \\
\hline
\end{tabular}

QUADRO 3. Tecnologias de Informação e Comunicação usadas como materiais didáticos desenvolvidos na disciplina "Prevenção de agravos, Promoção da Saúde e Redução de Vulnerabilidade: evolução histórica dos conceitos e oportunidades de práticas interprofissionais", em 2012 e 2013

\begin{tabular}{|l|l|l|l|l|}
\hline Ano & TIC & Finalidade da abordagem de ensino & $\begin{array}{l}\text { Ambiente de } \\
\text { uso }\end{array}$ & $\begin{array}{l}\text { Natureza da } \\
\text { atividade }\end{array}$ \\
\hline $\begin{array}{l}2012 \text { a } \\
2013\end{array}$ & Dropbox ${ }^{\circledR}$ & $\begin{array}{l}\text { Oferecer acesso a literatura obrigatória e sugerida } \\
\text { pelo corpo docente e criar espaço para sugestão de } \\
\text { outras referências pelos estudantes. }\end{array}$ & Virtual & Obrigatória \\
\hline $\begin{array}{l}2012 \text { a } \\
2013\end{array}$ & $\begin{array}{l}\text { Apresentação usando } \\
\text { Prezi® }\end{array}$ & $\begin{array}{l}\text { Apresentar de maneira não linear o percurso viven- } \\
\text { ciado ao longo da disciplina. }\end{array}$ & Em sala de aula & $\begin{array}{l}\text { Desenvolvida } \\
\text { pelo professor }\end{array}$ \\
\hline 2013 & $\begin{array}{l}\text { Nuvem de palavras } \\
\text { criadas no Wordle } \mathbb{R}\end{array}$ & $\begin{array}{l}\text { Compor de forma estética, o conteúdo identificado } \\
\text { sobre as expectativas dos participantes da disciplina, } \\
\text { de forma a destacar no tamanho das palavras, as } \\
\text { ideias mais frequentes. }\end{array}$ & Em sala de aula & $\begin{array}{l}\text { Desenvolvida } \\
\text { pelo professor }\end{array}$ \\
\hline 2013 & $\begin{array}{l}\text { Mapas conceituais } \\
\text { elaborados no CMAP } \\
\text { tools } \mathbb{}\end{array}$ & $\begin{array}{l}\text { Avaliar o conhecimento adquirido na forma de } \\
\text { construção de Mapa Conceitual Semi-estruturado, } \\
\text { em dois momentos: a) construção individual e b) } \\
\text { discussão e reconstrução em grupo interprofissional. }\end{array}$ & Em sala de aula & Obrigatória \\
\hline
\end{tabular}

QUADRO 4. Tecnologias de Informação e Comunicação usadas como materiais didáticos desenvolvidos na disciplina "Educação pela saúde na infância e adolescência”, na Escola de Enfermagem, Universidade de Costa Rica

\begin{tabular}{|c|c|c|c|c|}
\hline Ano & TIC & Finalidade da abordagem de ensino & $\begin{array}{l}\text { Ambiente de } \\
\text { uso }\end{array}$ & $\begin{array}{l}\text { Natureza da } \\
\text { atividade }\end{array}$ \\
\hline \multirow{4}{*}{$\begin{array}{l}2005 \\
\text { até } \\
2013\end{array}$} & $\begin{array}{l}\text { Sala de Aula Virtual, no } \\
\text { ambiente Moodle }{ }^{\circledR}\end{array}$ & $\begin{array}{l}\text { Oferecer um espaço aos estudantes para discutir, } \\
\text { conhecer os trabalhos de outros, procurar textos, } \\
\text { enviar trabalhos, fazer link aos e-portfolios }\end{array}$ & $\begin{array}{l}\text { Virtual ou Sala } \\
\text { de aula }\end{array}$ & $\begin{array}{l}\text { Obrigatória ou } \\
\text { optativa }\end{array}$ \\
\hline & $\begin{array}{l}\text { E-portfolio } \\
\text { (WordPress } \AA \text {, Blogspot } \AA \text {, } \\
\text { Evernote } \AA \text { ) }\end{array}$ & $\begin{array}{l}\text { Mostrar o relato reflexivo de experiências, os } \\
\text { avanços bem como evidenciar a construção de } \\
\text { conhecimento tanto individual quanto em grupo }\end{array}$ & Virtual & Obrigatória \\
\hline & $\begin{array}{l}\text { Mapas conceituais; } \\
\text { CMAP tools } \AA\end{array}$ & $\begin{array}{l}\text { Facilitar a construção de conceitos e ainda a } \\
\text { inter-relação entre os mesmos. Registrar os pro- } \\
\text { cessos cognitivos de uma situação ou tema }\end{array}$ & $\begin{array}{l}\text { Virtual para fazer } \\
\text { e Sala de aula } \\
\text { para discutir }\end{array}$ & Obrigatória \\
\hline & Vídeos & $\begin{array}{l}\text { Seleção feita pelos estudantes para complemen- } \\
\text { tar a construção dos saberes }\end{array}$ & $\begin{array}{l}\text { Virtual ou Sala } \\
\text { de aula }\end{array}$ & Optativa \\
\hline
\end{tabular}




\section{DISCUSSÃO}

As três experiências utilizaram diferentes formas de TIC, privilegiando ferramentas gratuitas e de acesso livre. A Comissão Global para a educação dos profissionais da saúde no século 21 destaca o potencial de tais ferramentas ${ }^{13}$. Wipfli et al. ${ }^{14}$ reforçam que é preciso experimentar e compartilhar experiências que utilizem TIC, para o desenvolvimento de propostas educacionais efetivas. Os autores concluem que a oportunidade das TIC traz desafios complexos para docentes e estudantes. Nesta direção, as experiências acadêmicas analisadas retrataram o esforço de professores e estudantes na formação em promoção da saúde.

Tais TIC podem ser agrupadas em três categorias, de acordo com Sargeant et al. ${ }^{15}$. A primeira envolveu a apresentação do conteúdo teórico, tanto em sala de aula quanto no ambiente virtual (acesso aos materiais e referências via internet, via blog). Observou-se também a incorporação de TIC que estimulavam a interação do estudante com o conteúdo (palavras cruzadas e e-portfolio). Por fim, foram propostos espaços virtuais de interação entre os estudantes (recursos do Moodle ${ }^{\circledR}$ e grupo de e-mails).

Fica evidenciada a interlocução de modelos ora centrado no conteúdo, ora no estudante. Tal diálogo entre as formas tradicionais e as propostas inovadoras é essencial para a construção e reconstrução da novidade na prática ${ }^{16}$. Entretanto, como proposto por D'Amour e Oandasan ${ }^{17}$, é possível traçar um paralelo entre o campo da formação e o campo das práticas. As autoras defendem um modelo de ensino-aprendizagem "estudante-centrado", que acompanhe um modelo de atenção à saúde "usuário- centrado" em contrapartida ao modelo "procedimentocentrado".

Portanto, é preciso lembrar que o uso das TIC deve ser significativo para os atores envolvidos no processo de ensino-aprendizagem. É reconhecida a insuficiência de tais ferramentas como mudança pedagógica isolada. Cavalcante e Vasconcellos ${ }^{18}$ enriquecem a discussão e reforçam o alerta feita por Belloni ${ }^{19}$ apud Cavalcante e Vasconcellos ${ }^{18}$ sobre o risco da incorporação acelerada e acrítica. Nesta direção, os autores apresentam duas revisões, a primeira sobre a incorporação de TICs tanto na saúde quanto na educação. Já a segunda trata de conceitos tecnológicos, aplicáveis nas duas áreas. Os autores valorizam a troca de conteúdos, a reutilização de materiais educativos e a interdisciplinaridade

Alinhada ao referencial teórico do campo da promoção da saúde, é preciso que o processo de formação sobre o tema busque o desenvolvimento de profissionais cidadãos, isto é, de profissionais comprometidos com as necessidades e expectativas sociais ${ }^{15}$. A promoção da saúde é tecida de atividades educativas contínuas, sendo essencial aqui considerar a educação uma prática social, voltada para a formação da pessoa na sua totalidade, onde há uma ação intencional, com vista ao crescimento e formação pessoal.

Conclui-se que, frente as possibilidades pedagógicas em promoção da saúde relacionadas às TIC, é necessário e prudente o desenvolvimento de novas estratégias de ensino, pesquisa e extensão que incorporem os elementos de uma aprendizagem centrada no próprio estudante e o bom uso de tecnologias digitais em prol de sua formação contínua.

\section{REFERÊNCIAS}

1. Levy P. Cybercultura. São Paulo: Editora 34; 2010.

2. Ebert CRC. O ensino semi-presencial como resposta às crescentes necessidades de educação permanente. Educar. 2003;(21):82-98. Disponível em: http://ojs.c3sl.ufpr.br/ojs2/ index.php/educar/article/view/2124/1776

3. Ricardo EJ,organizador. Educação corporativa e educação à distância. Rio de Janeiro: Qualitymark; 2005.

4. Carbonaro M, King S, Taylor E, Satzinger F, Snart F, Drummond J. Integration of e-learning technologies in an interprofessional health science course. MedTeach. 2008;30:25-33. doi: 10.1080/01421590701753450.

5. Macdonald J. Blended learning and online tutoring. Br J Educ Technol. 2008;39(6):1147. http://onlinelibrary.wiley.com/ doi/10.1111/j.1467-8535.2008.00908_22.x/pdf

6. Pulman A, Scammell J, Martin M. Enabling interprofessional education: the role of technology to enhance learning. Nurse Educ Today. 2009;29:232-9. doi: 10.1016/j. nedt.2008.08.012.
7. Souza AMA, Galvão EA, Santos I, Roschke MA. Processos educativos nos serviços de saúde. (Série de desenvolvimento de recursos humanos parte 1). Brasília: OPAS; 1991. Disponível em: https://www.nescon.medicina.ufmg.br/ biblioteca/imagem/0182.pdf

8. Brasil. Ministério da Saúde. Fundação Osvaldo Cruz. Promoção da Saúde. Carta de Ottawa, Declaração de Adelaide, Declaração de Sundswall, declaração de Santa-Fé de Bogotá, Declaração de Jacarta, Rede de Mega países e Declaração do México. Trad. Le Fonseca e R Fernandez. Brasília; 2001.

9. World Health Organization. Bangkok charter for health promotion in the a globalized world. Geneve: WHO; 2005 [cited 2010 July 20]. Available from: http://www.who.int/ healthpromotion/conferences/6gchp/hpr_050829_\%20BCHP. pdf

10. Boet S. Sharma S. Goldman J, Reeves S. Review article: Medical education research: an overview of methods. Can J Anesth. 2012;59:159-70. doi: 10.1007/s12630-011-9635-y. 
11. Frye AW. Hemmer PA. Program evaluation models and related theories: AMEE Guide No. 67. Medical Teach. 2012;34:e288e299. doi: 10.3109/0142159X.2012.668637.

12. Taub A, Allegrante JP, Barry MM, Sakagami K. Perspectives on Terminology and Conceptual and Professional Issues in Health Education and Health Promotion Credentialing. Health Educ Behav. 2009;36:439. doi: 10.1177/1090198109333826

13. Frenk J, Chen L, Bhutta ZA, Cohen J, Crisp N, Evans T, Finenberg $\mathrm{H}$, et al. Health professionals for a new century: transforming education to strengthen health systems in an interdependent world. Lancet. 2010;376(9756):1923-58. doi: 10.1016/S0140-6736(10)61854-5.

14. Wipfli H, Press DJ, Kuhn V. Global health education: a pilot in trans-disciplinary, digital instruction. Glob Health Action. 2013;6:1-7. doi: 10.3402/gha.v6i0.20747.

15. Sargeant J, Curran V et al. Interactive on-line continuing medical educational: physicians' perceptions and experiences.
J Contin Educ Health Prof. 2004;24:227-36. doi: 10.1002/ chp. 1340240406

16. Guimarães DA, Silva ES. Formação em ciências da saúde: diálogos em saúde coletiva e a educação para a cidadania. Ciên Saúde Coletiva. 2010;15(5):2551-62. http://dx.doi. org/10.1590/S1413-81232010000500029

17. D'Amour E. Oandasan I. Interprofessionality as the field of interprofissional practice and interprofessional education: An emerging concept. J Interprofessional Care. 2005;S1: 8-20. doi:10.1080/13561820500081604

18. Cavalcante MTL, Vasconcellos MM. Tecnologia de informação para a educação na saúde: duas revisões e uma proposta. Ciên Saúde Coletiva. 2007;12(3):611-22. http:// dx.doi.org/10.1590/S1413-81232007000300011

19. Belloni ML. Ensaio sobre a educação à distância no Brasil. Educ Sociedade. 2002;78: 117-42. Disponível em: http:// www.scielo.br/pdf/es/v23n78/a08v2378.pdf 